$4 v+1,4 v+3$. When $n=4 v+2$, no odd branch can exist. Exceptional are the cases when $n=3,4,5$, the maximum number of odd branches being $1,2,3$, respectively. Then, by applying Ábel's theorem for elliptic functions, he proves, for every value of $n$, the existence of curves with the maximum number of real odd branches.

Worcester, Mass., April 5, 1892.

L. S. Hulburt.

\title{
FINAL FORMULAS FOR THE ALGEBRAIC SOLUTION OF QUARTIC EQUATIONS.*
}

BY MANSFIELD MERRIMAN, PH.D.

I. Finac formulas for the algebraic solution of quadratic and cubic equations are well known. Such formulas exhibit the roots in their true typical forms, and lead to ready and exact numerical solutions whenever the given equations do not fall under the irreducible case. But for the quartic, or biquadratic, equation the books on algebra do not give similar final formulas. The solution of the quartic has been known since 1540, and numerous methods have been dednced for its algebraic resolution, yet in no caso does this appear to have been completed in final practical shape. It is the object of this paper to state the final solution in the form of definite formulas.

II. The expression of the roots of the quartic is easily made in terms of the roots of a resolvent cubic, and the cubic itself is solved without difficulty. Yet great practical difficulty exists in treating a numerical equation on account of the presence of imaginaries in the roots of the resolvent. Witness the following example which is generally given to illustrate the method in connection with Euler's resolvent:

"Let it be required to determine the roots of the biquadratic equation,

$$
x^{4}-25 x^{2}+60 x-36=0 .
$$

By comparing this with the general form the cubic equation to be resolved is,

$$
y^{3}-50 y^{2}+729 y-3600=0
$$

* Abstract of a paper presented to the Sociery at the meeting of May 7, 1892. 
the roots of which are found, by the rules for cubics, to be 9 , 16 and 25 , so that $\sqrt{y_{1}}=3, \sqrt{y_{2}}=4$, and $\sqrt{y_{3}}=5$. Therefore, since the coefficient of $x$ is positive,

$$
\begin{aligned}
& x_{1}=\frac{1}{2}(-3-4-5)=-6 \\
& x_{2}=\frac{1}{2}(-3+4+5)=+3 \\
& x_{3}=\frac{1}{2}(+3-4+5)=+2 \\
& x_{4}=\frac{1}{2}(+3+4-5)=+1
\end{aligned}
$$

which are the four roots of the proposed equation."

III. Now all that can be said of this numerical work is that it is a verifying instance. It is not an algebraic solution in any sense of the word, for, as both the quartic and its cubic resolvent have real roots, this is the irreducible case where the numerical solution fails. In Euler's Algebra, 17\%4, where this example was first given, the roots of the cubic are obtained by the use of trigonometrical tables, but in subsequent quotations it is usually merely stated that they are found " by the rules for cubics." This numerical example has certainly no place in the exemplification of the algebraic solution of the quartic equation, and yet it is so given in most mathematical dictionaries and it may also be seen in the article Algebra in the last edition of the Encyclopædia Britannica.

As this Bulletin is intended for historical and critical remarks rather than for original investigations it will not be well to here set forth the method whereby I have brought the solution into such shape as to produce final practical formulas. But the results may perhaps be allowed place, as their statement is very brief.

IV. The following are final formulas for the algebraic solution of the quartic equation,

$$
x^{4}+4 a x^{3}+6 b x^{2}+4 c x+d=0 .
$$

First, let $m$ and $n$ be determined by

$$
\begin{aligned}
& m=a^{2} d-2 a b c+b^{3}-b d+c^{2} \\
& n=\left(b^{2}+\frac{1}{3} d-\frac{4}{3} a c\right)^{3} .
\end{aligned}
$$

Secondly, let $s$ and $t$ be found from

$$
\begin{aligned}
& s=\frac{1}{2}\left(m+\sqrt{m^{2}-n}\right)^{\frac{1}{3}} \\
& t=\frac{1}{2}\left(m-\sqrt{m^{2}-n}\right)^{\frac{3}{3}}
\end{aligned}
$$


Thirdly, let $u, v$, and $w$ be derived by,

$$
\begin{aligned}
& u=\left(a^{2}-b\right)-(s+t) \\
& v=2\left(a^{2}-b\right)-(s+t) \\
& w=v^{2}+3(s-t)^{2} .
\end{aligned}
$$

Then the four roots of the given quartic are expressed by the formulas,

$$
\begin{aligned}
& x_{1}=-a+\sqrt{u}+\sqrt{v+\sqrt{w}} \\
& x_{2}=-a+\sqrt{u}-\sqrt{v+\sqrt{w}} \\
& x_{3}=-a-\sqrt{u}+\sqrt{v-\sqrt{w}} \\
& x_{4}=-a-\sqrt{u}-\sqrt{v-\sqrt{w}}
\end{aligned}
$$

in which the signs before the square roots are to be used as written provided $2 a^{3}-3 a b+c$ is negative, but if this is positive all radicals except $\sqrt{w}$ are to be reversed in sign.

V. As a numerical example, let the equation to be solved be the complete quartic,

$$
x^{4}-8 x^{3}-10 x^{2}+56 x+192=0 .
$$

Here, by comparing the coefficients with the given form,

$$
a=-2, \quad b=-\frac{5}{3}, c=+14, d=+192 .
$$

From these are first computed,

$$
m=\frac{32023}{27}, \quad n=\frac{822656953}{729},
$$

and next in order are found,

$$
s=5.983, \quad t=4.350 .
$$

Accordingly $s+t$ is 10.333 , and $s-t$ is 1.633 , and then

$$
u=16, \quad v=1, \quad w=9 .
$$

Now as $2 a^{3}-3 a b+c$ has a negative value, the formulas give

$$
x_{1}=2+4+\sqrt{1+3}=+8
$$




$$
\begin{aligned}
& x_{2}=2+4-\sqrt{1+3}=+4 \\
& x_{3}=2-4+\sqrt{1-3}=-2+\sqrt{-2} \\
& x_{4}=2-4-\sqrt{1-3}=-2-\sqrt{-2}
\end{aligned}
$$

which are the simplest expressions for the four roots.

As a second example let the proposed quartic equation be $x^{4}+7 x+6=0$. Here $a=0, b=0, c=\frac{7}{4}$, and $d=6$. Then $m=\frac{49}{1}$ and $n=8$. Next in order, $s=0.8091$ and $t=0.6180$, whence $u=+1.42 \%, v=-1.42 \%$ and $w=2.146$. Now, $c$ being positive, the roots are

$$
\begin{aligned}
& x_{1}=-1.194-\sqrt{-1.427+1.465}=-1.388 \\
& x_{2}=-1.194+\sqrt{-1.424+1.465}=-1.000 \\
& x_{3}=+1.194-\sqrt{-1.427-1.465} \\
& x_{4}=+1.194+\sqrt{-1.427-1.465}
\end{aligned}
$$

which closely satisfy the given equation.

VI. The above formulas for the algebraic solution of the quartic equation are final in the sense that, like those so well known for the quadratic and cubic, they exhibit true symbolic representations of the roots in terms of the given coefficients, and that they are not capable of further essential simplification. They furnish the means of the discussion of all the circumstances concerning the occurrence of equal roots in the quartic, as well as of cases where the roots are connected by a known relation. They will be found to embrace the solution of all special and critical cases. For instance, applied to the binomial $x^{4}-1=0$ they give the roots $x_{1}=+1, x_{2}=-1$, $x_{3}=+\sqrt{-1}$ and $x_{4}=-\sqrt{-1}$. Again, if applied to the form $x^{4}+6 b x^{2}+d=0$, they give the same solution as that by quadratics, for $u$ becomes zero, $v$ becomes $-3 b$ and $w$ reduces to $9 b^{2}-d$. Lastly, they furnish ready and exact numerical solutions whenever the proposed equation has two real and two imaginary roots, or when two or more roots are equal. If there be either four unequal real roots or four unequal imaginary roots, the irreducible case arises where $m^{2}-n$ becomes negative, and the formulas, although correctly representing the roots, fail to furnish numerical solutions in as simple forms as desired.

Lehigh UnIVersity, March, 1892. 